\title{
Global Lie symmetries of a system of Schrödinger equations and the oscillator representation
}

\author{
Markus Hunziker, Mark R. Sepanski, and Ronald J. \\ Stanke
}




\title{
GLOBAL LIE SYMMETRIES OF A SYSTEM OF SCHRÖDINGER EQUATIONS AND THE OSCILLATOR REPRESENTATION
}

\author{
MARKUS HUNZIKER, MARK R. SEPANSKI, AND RONALD J. STANKE
}

\begin{abstract}
We realize the oscillator representation of the metaplectic group $\operatorname{Mp}(n)$ in the space of solutions to a system of Schrödingier type equations on $\mathbb{R}^{n} \times \operatorname{Sym}(n, \mathbb{R})$. Our realization has particularly simple intertwining maps to the realizations given by Kashiwara and Vergne.
\end{abstract}

2000 Mathematics Subject Classification: Primary 22E45; Secondary 22E70

Keywords: Oscillator representation, metaplectic group, Jacobi group, Schrödinger equation

\section{INTRODUCTION}

The origin of Lie theory lies in the symmetry analysis of systems of partial differential equations. These ideas have proved to be one of the most fruitful tools in the study of representation theory. Generalizing work in $[21,22]$ and using techniques similar to those found in [14], this paper employs Lie symmetry analysis to study the system of partial differential equations

$$
\begin{aligned}
4 s \partial_{t_{i i}} f(x, t)+\partial_{x_{i}}^{2} f(x, t) & =0, & & 1 \leq i \leq n \\
2 s \partial_{t_{i j}} f(x, t)+\partial_{x_{i}} \partial_{x_{j}} f(x, t) & =0, & & 1 \leq i<j \leq n
\end{aligned}
$$

with $s \in i \mathbb{R}^{\times}$. Here, $x=\left(x_{i}\right)$ and $t=\left(t_{i j}\right)$ are the canonical coordinates on $\mathbb{R}^{n}$ and the space of real symmetric matrices $\operatorname{Sym}(n, \mathbb{R})$, respectively. Lie's prolongation method calculates the infinitesimal symmetries of system $\left(^{*}\right)$ to be the Jacobi Lie algebra $\mathfrak{g}=\mathfrak{s p}(n, \mathbb{R}) \ltimes \mathfrak{h}_{2 n+1}$, where $\mathfrak{s p}(n, \mathbb{R})$ is the symplectic Lie algebra on $\mathbb{R}^{2 n}$ and $\mathfrak{h}_{2 n+1}$ is the $(2 n+1)$-dimensional Heisenberg Lie algebra, plus an infinite dimensional Lie algebra reflecting the fact that system $(*)$ is linear. In particular, the space of all complex-valued functions $f \in \mathcal{C}^{\infty}\left(\mathbb{R}^{n} \times \operatorname{Sym}(n, \mathbb{R})\right)$ satisfying system $\left(^{*}\right)$ carries a representation of $\mathfrak{g}$. The $\mathfrak{g}$-action on $\mathcal{C}^{\infty}\left(\mathbb{R}^{n} \times \operatorname{Sym}(n, \mathbb{R})\right)$ does not exponentiate to a global action of the Jacobi group $G^{J}=\operatorname{Sp}(n, \mathbb{R}) \ltimes H_{2 n+1}$ or any cover group. However, as we will explain below, there are canonical $\mathfrak{g}$-invariant subspaces $d_{ \pm}^{\prime} \subseteq \mathcal{C}^{\infty}\left(\mathbb{R}^{n} \times \operatorname{Sym}(n, \mathbb{R})\right)$ such that the $\mathfrak{g}$-action on $d_{ \pm}^{\prime}$ does exponentiate to a global action of the group $G=\operatorname{Mp}(n) \ltimes H_{2 n+1}$, where $\mathrm{Mp}(n)$ is the metaplectic group, i. e., the double cover of $\operatorname{Sp}(n, \mathbb{R})$. The end result of our analysis shows that 
the space of solutions to system $(*)$ in $d_{ \pm}^{\prime}$ gives a realization of the oscillator representation (or its dual depending on the sign of $\sigma$ ) of $\mathrm{Mp}(n)$ with particularly simple intertwining maps to two realizations given by Kashiwara and Vergne in [16].

One of the most ubiquitous representations found in the literature is the oscillator representation $\omega$, often called the metaplectic or Segal-Shale-Weil representation, a genuine representation of $G$. We mention only briefly some of the settings in which this representation appears and refer the reader to [4] for a thorough development.

From classical number theory, the invariance properties of Jacobi theta functions [8] are found by lifting such functions to $G^{J}$. This lift, in turn, utilizes the oscillator representation [5]. A complete treatment of theta functions appears in [15] and many more results demonstrating the interplay between $\omega$ and aspects of number theory can be found in $[17,18,27]$.

The quantization procedure in theoretical physics associates classical geometric systems to quantum mechanical systems and is very well studied ([11,24-26,28], and [1]). For example, the oscillator representation arises in quantum mechanics when one quantizes a single particle structure [20]. The representation $\omega$ is constructed and then used to establish results about the inducibility of a field automorphism by a unitary operator in all quantizations [23]. Another application of $\omega$ appears in quantum optics. In [2], the tensor product of $\omega$ with discrete series representations of $\mathrm{SU}(1,1)$ admits squeezed coherent states. The broader role that $\omega$ plays in physics can be found in $[7,12]$.

In the representation theory, the oscillator representation is used to construct other important representations. For instance, the representations of $G^{J}(n=1)$ with nontrivial central character are realized as products of representations of $\mathrm{Mp}(1)$ and the oscillator representation [5]. In the well-known article [16], the $k$-fold tensor product $\otimes_{k} \omega$ is decomposed into irreducible unitary representations. First conjectured by Kashiwara and Vergne and later proved by Enright and Parthasarathy [9], all irreducible unitary highest weight representations for which the Verma module $N(\lambda+\rho)$ is reducible (i. e., $\lambda$ is a reduction point) are found in $\otimes_{k} \omega$ for some $k$. In a similar vein, it is shown in [13] that every genuine discrete series representation of $\operatorname{Mp}(n)$ appears in $\left(\otimes_{k} \omega\right) \otimes\left(\otimes_{m} \omega^{*}\right)$, for some $k$ and $m$. Finally, if $F$ is a finite field, irreducible representations of GL $(2, F)$ can be constructed by using the Weil representation ([6]), the restriction of $\omega$ to $\operatorname{SL}(2, F)$. For $F$, a non-Archimedean local field, the same is true of many supercuspidal irreducible representations of GL(2,F).

Given the manifold applications of $\omega$, it may be helpful to identify some canonical realizations. A standard realization of $\omega$ arises via the Stone - von Neumann theorem as an intertwining operator between equivalent irreducible unitary representations of $H_{2 n+1}$ on $L^{2}\left(\mathbb{R}^{n}\right)$ ([10] and, in more generality, [27]). A second realization is the Fock model, where $\omega$ is realized as an integral operator on a reproducing kernel space. Motivated by Lie's prolongation method ([19]), we induce from a subgroup of $G$ and use a system of Schrödinger type equations to find a subspace on which 
the action is irreducible. In [3], a reproducing space of holomorphic functions on $\operatorname{Sp}(n, \mathbb{R}) / U(n) \times U(n)$ is shown to satisfy analogous differential equations (if one replaces real with complex differentiation), but no unitary action on that space is provided.

We now give an overview of our results. Kashiwara and Vergne give an embedding of the tensor product of the oscillator representation into a subspace of sections of vector bundles over Siegel upper half-space, $\mathfrak{H}_{n}$, and also into a subspace of certain principal series representations. For instance, in the case of the even part of the oscillator representation, they construct the maps

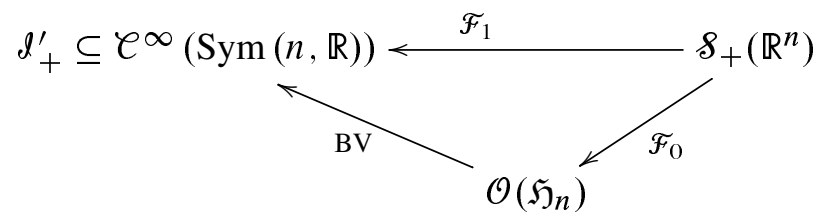

where $\delta\left(\mathbb{R}^{n}\right)$ denotes the set of Schwartz functions on $\mathbb{R}^{n}, \delta_{+}\left(\mathbb{R}^{n}\right)$ denotes the even Schwartz functions, $d_{+}^{\prime}$ denotes the image of $\delta_{+}\left(\mathbb{R}^{n}\right)$ under the map $\widetilde{F}_{1}=$ $\mathrm{BV} \circ \widetilde{F}_{0}$ (with $\mathcal{C}^{\infty}(\operatorname{Sym}(n, \mathbb{R}))$ being the noncompact picture of a certain principal series representation of the metaplectic group $\operatorname{Mp}(n)$ ), and the maps are given by the equalities $(\mathrm{BV} \Psi)(t)=\lim _{Y \rightarrow 0^{+}} \Psi(t+i Y)$ and

$$
\begin{aligned}
& \left(\mathcal{F}_{0} \psi\right)(Z)=\int_{\mathbb{R}^{n}} \psi(\xi) e^{\frac{i}{2} \xi Z \xi^{T}} d \xi, \\
& \left(\mathcal{F}_{1} \psi\right)(t)=\int_{\mathbb{R}^{n}} \psi(\xi) e^{\frac{i}{2} \xi t \xi^{T}} d \xi,
\end{aligned}
$$

where $\mathbb{R}^{n}$ is identified with $M_{1 \times n}(\mathbb{R}), \psi \in \mathcal{S}_{+}\left(\mathbb{R}^{n}\right), Z \in \mathfrak{H}_{n}, t \in \operatorname{Sym}(n, \mathbb{R}), \Psi \in$ $\operatorname{Im}\left(\widetilde{F}_{0}\right) \subseteq \mathcal{O}\left(\mathfrak{H}_{n}\right)$, and $\lim _{Y \rightarrow 0^{+}}$denotes the limit as $Y \rightarrow 0$ with $Y \in \operatorname{Sym}(n, \mathbb{R})$ and $Y>0$.

Turning to our realization in a special case, we have a commutative diagram

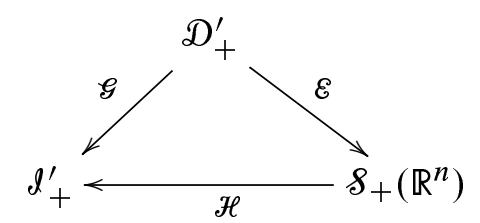

where $\mathscr{D}_{+}^{\prime} \subseteq \mathcal{C}^{\infty}\left(\mathbb{R}^{n} \times \operatorname{Sym}(n, \mathbb{R})\right)$ is a subset of smooth functions (coming from an induced representation via the noncompact picture) $f$ in the kernel of the operators $4 \pi^{2} i \partial_{t_{i j}}-\partial_{x_{i}} \partial_{x_{j}}, i \neq j$, and $8 \pi^{2} i \partial_{t_{i i}}-\partial_{x_{i}}^{2}$ with $f(\cdot, t) \in \mathcal{S}_{+}\left(\mathbb{R}^{n}\right)$ for each $t \in$ $\operatorname{Sym}(n, \mathbb{R})$ and $d_{+}^{\prime} \subseteq \mathcal{C}^{\infty}(\operatorname{Sym}(n, \mathbb{R}))$ consisting of the set of Fourier transforms of Schwartz functions pulled back as measures on $\left\{-y^{T} y \mid y \in \mathbb{R}^{n}\right\} \subseteq \operatorname{Sym}(n, \mathbb{R})$. The maps $\mathscr{E}$ and $\mathcal{E}$ are particularly simple:

$$
(\varepsilon f)(x)=\hat{f}(x, 0) \text { and }(\mathscr{E} f)(t)=f(0, t)
$$


(with the Fourier transform given by $\left.\hat{f}(\xi)=\int_{\mathbb{R}^{n}} f(x) e^{-2 \pi i \xi x^{T}} d x\right)$. There is an explicit integral formula for $\varepsilon^{-1}$ given by

$$
\left(\mathcal{E}^{-1} \psi\right)(x, t)=\int_{\mathbb{R}^{n}} f(\xi) e^{\frac{i}{2} \xi t \xi^{T}} e^{2 \pi i \xi x^{T}} d \xi,
$$

which gives rise to a formula for $\mathscr{H}=\mathscr{F}_{1}$ as $(\mathscr{H} \psi)(t)=\int_{\mathbb{R}^{n}} f(\xi) e^{\frac{i}{2} \xi t \xi \xi^{T}} d \xi$. An inverse for $\mathcal{E}$ can be given by viewing elements of $\mathscr{d}_{+}^{\prime}$ as tempered distributions on $\operatorname{Sym}(n, \mathbb{R})$, applying a Fourier transform and taking a limit using approximations to a $\delta$-function.

The highest weight vector in $\mathscr{D}_{+}^{\prime}$ is the function $f_{+} \in \mathcal{C}^{\infty}\left(\mathbb{R}^{n} \times \operatorname{Sym}(n, \mathbb{R})\right)$ defined as

$$
f_{+}(x, t)=\operatorname{det}\left(I_{n}-i t\right)^{-\frac{1}{2}} e^{-2 \pi^{2} x\left(I_{n}-i t\right)^{-1} x^{T}} .
$$

The corresponding vector in $d_{+}^{\prime}$ is $f_{+}(0, t)=\operatorname{det}\left(I_{n}-i t\right)^{-\frac{1}{2}}$ and in $\gamma_{+}\left(\mathbb{R}^{n}\right)$ is

$$
\widehat{f}_{+}(\xi, 0)=(2 \pi)^{-\frac{n}{2}} e^{-\frac{1}{2}\|\xi\|^{2}} .
$$

Note that the choice of, say, $s=2 \pi^{2} i$ gives rise to the dual representation and Schrödinger-like partial differential operators with the lowest weight representations.

The above commutative diagram fits on top of the Kashiwara-Vergne picture to give the following combined commutative diagram.

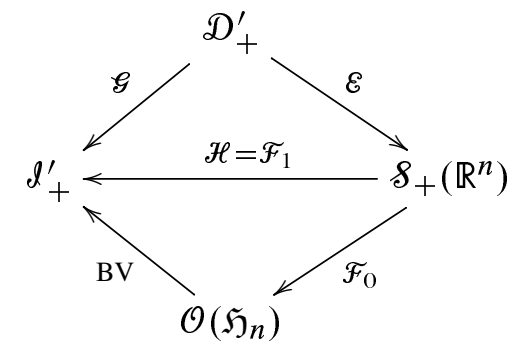

There is a similar picture for the odd part of the oscillator representation that fits in with the Kashiwara-Vergne realization in an analogous way. The main change is that $\mathscr{G}$ is replaced by the operator $\left(\mathscr{G}_{n} f\right)(t)=\nabla_{\mathbb{R}^{n}} f(0, t)$ and $\mathscr{H}$ by the operator

$$
\left(\mathscr{H}_{n} f\right)(t)=\left.\nabla\left(\int_{\mathbb{R}^{n}} f(\xi) e^{\frac{i}{2} \xi t \xi^{T}} e^{2 \pi i \xi x^{T}} d \xi\right)\right|_{x=0} .
$$

\section{A DOUBLE COVER OF THE JACOBI GROUP}

With respect to the standard symplectic form $J_{n+1}=\left(\begin{array}{cc}0 & -I_{n+1} \\ I_{n+1} & 0\end{array}\right)$, the Jacobi group is

$$
G^{J}=\operatorname{Sp}(n+1, \mathbb{R}) \cap\left\{\left(\begin{array}{cc}
* & * \\
0_{1 \times(2 n+1)} & 1
\end{array}\right)\right\} \cong \operatorname{Sp}(n, \mathbb{R}) \ltimes H_{2 n+1},
$$


where $H_{2 n+1}$ is the $2 n+1$ dimensional real Heisenberg Lie group. It has Lie algebra $\mathfrak{g} \cong \mathfrak{s p}(n, \mathbb{R}) \ltimes \mathfrak{h}_{2 n+1}$, where $\mathfrak{h}_{2 n+1}$ is the $2 n+1$ dimensional real Heisenberg Lie algebra. We write $\mathfrak{H}_{n}$ for the Siegel upper half-space consisting of the set of $Z=X+i Y$ with $X, Y \in \operatorname{Sym}(n, \mathbb{R})$ and $Y>0$ (positive definite), which carries a transitive action by $\operatorname{Sp}(n, \mathbb{R})$ by linear fractional transformations, $g \cdot Z=$ $(A Z+B)(C Z+D)^{-1}$ where $g=\left(\begin{array}{ll}A & B \\ C & D\end{array}\right) \in \operatorname{Sp}(n, \mathbb{R})$. Note that the stabilizer of $i I_{n}$ in $\operatorname{Sp}(n, \mathbb{R})$ is a maximal compact subgroup $K \cong U(n)$.

The main object of study is the double cover of $G^{J}$,

$$
G=\operatorname{Mp}(n) \ltimes H_{2 n+1} .
$$

Here, the action of $\operatorname{Mp}(n)$ on $H_{2 n+1}$ factors through its projection to $\operatorname{Sp}(n, \mathbb{R})$ and we realize the metaplectic group $\operatorname{Mp}(n)$ as the set of pairs $(g, \varepsilon)$ with $g \in \operatorname{Sp}(n, \mathbb{R})$ and smooth $\varepsilon: \mathfrak{H}_{n} \rightarrow \mathbb{C}$ satisfying $\varepsilon(Z)^{2}=\operatorname{det}(C Z+D)$. The group law on $\operatorname{Mp}(n)$ is given by $\left(g_{1}, \varepsilon_{1}\right) \cdot\left(g_{2}, \varepsilon_{2}\right)=\left(g_{1} g_{2}, Z \rightarrow \varepsilon_{1}\left(g_{2} \cdot Z\right) \varepsilon_{2}(Z)\right)$, and to be explicit, the group law on $\operatorname{Mp}(n) \ltimes H_{2 n+1}$ is given by

$$
\left(\left(g_{1}, \varepsilon_{1}\right), h_{1}\right) \cdot\left(\left(g_{2}, \varepsilon_{2}\right), h_{2}\right)=\left(\left(g_{1}, \varepsilon_{1}\right) \cdot\left(g_{2}, \varepsilon_{2}\right), g_{2}^{-1} h_{1} g_{2} h_{2}\right) .
$$

We also need to extend the definition of $\varepsilon$ from $\mathfrak{H}_{n}$ to $\operatorname{Sym}(n, \mathbb{R})$ almost everywhere. For this, let $\varepsilon: \operatorname{Sym}(n, \mathbb{R}) \rightarrow \mathbb{C}$ be given by $\varepsilon(X)=\lim _{Y \rightarrow 0^{+}} \varepsilon(X+i Y)$ (here $Y \rightarrow 0^{+}$denotes $Y \rightarrow 0$ with $\left.Y>0\right)$, which will be defined when $\operatorname{det}(C X+D) \neq$ 0 . In particular, it is easy to see that $\varepsilon(X)$ exists and is given by

$$
\varepsilon(X)=i^{l} \sqrt{|\operatorname{det}(C X+D)|},
$$

where $l=l(\varepsilon, X)$ is one of the two choices (determined precisely by $\varepsilon$ and $X$ ) of $l \in \mathbb{Z}_{4}$ for which $(-1)^{l}=\operatorname{sgn}(\operatorname{det}(C X+D))$. In a similar manner, we extend to an almost everywhere action of $\operatorname{Sp}(n, \mathbb{R})$ on $\operatorname{Sym}(n, \mathbb{R})$ given by $g \cdot X=$ $(A X+B)(C X+D)^{-1}$ for $X \in \operatorname{Sym}(n, \mathbb{R})$ when $\operatorname{det}(C X+D) \neq 0$.

\section{THE INDUCED REPRESENTATIONS}

Consider the subalgebra $\overline{\mathfrak{p}}$ of $\mathfrak{g}$ given as the semidirect product of the maximal parabolic subalgebra $\overline{\mathfrak{p}}_{\mathfrak{s p}}$ of $\mathfrak{s p}(n, \mathbb{R})$ consisting of lower $n \times n$ block-diagaonal matrices and the maximal abelian subalgebra $\mathfrak{w}$ of $\mathfrak{h}_{2 n+1}$ isomorphic to $\mathbb{R}^{n} \times \mathbb{R}$ that is stabilized by $\overline{\mathfrak{p}}_{\mathfrak{s p}}$. Write the Langlands decomposition for $\overline{\mathfrak{p}}_{\mathfrak{s p}}$ as $\overline{\mathfrak{p}}_{\mathfrak{s p}}=\mathfrak{m a} \overline{\mathfrak{n}}$. Write $\bar{P}=M A \bar{N} \ltimes W$ for the corresponding groups. It turns out that $M \cong\{(A, c) \mid$ $\left.A \in G L(n, \mathbb{R}), \operatorname{det} A \in\{ \pm 1\}, c^{2}=\operatorname{det} A^{-1}\right\}$, so the component group $M / M_{0}$ is isomorphic to $\mathbb{Z}_{4}, A \cong \mathbb{R}^{+}, \bar{N} \cong \operatorname{Sym}(n, \mathbb{R})$, and $W \cong \mathbb{R}^{n+1}$.

For $q \in \mathbb{Z}$ (determined only mod 4), $r \in \mathbb{C}$, and $s \in \mathbb{C}$, we define a character $\chi_{q, r, s}: \bar{P} \rightarrow \mathbb{C}$ by $\chi_{q, r, s}(A, c)=c^{q}$ on $M, \chi_{q, r, s}(a)=a^{r n}$ on $A$, and $\chi_{q, r, s}$ is 
trivial on $W$ and $\bar{N}$. We study the induced representation

$$
\begin{aligned}
I(q, r, s) & =\operatorname{Ind}_{\bar{P}}^{G} \chi_{q, r, s} \\
& =\left\{\mathcal{C}^{\infty} \phi: G \rightarrow \mathbb{C} \mid \phi(g p)=\chi_{q, r, s}(p)^{-1} \phi(g) \text { for } g \in G, p \in \bar{P}\right\}
\end{aligned}
$$

with group action $(g \cdot \phi)\left(g^{\prime}\right)=\phi\left(g^{-1} g^{\prime}\right)$.

We will also have an opportunity to make use of two related induced representations of $\operatorname{Mp}(n)$. To this end, define a character and an $n$-dimensional representation of $M A \bar{N}, \chi_{q, r}: M A \bar{N} \rightarrow \mathbb{C}$ and $\pi_{q, r}: M A \bar{N} \rightarrow \operatorname{GL}(n, \mathbb{C})$, by $\chi_{q, r}=\left.\chi_{q, r, s}\right|_{M A \bar{N}}$ and $\pi_{q, r}((A, c) a \bar{n})=\chi_{q, r}((A, c) a \bar{n}) A^{-1}$. The associated induced representations are $I(q, r)=\operatorname{Ind}_{M A \bar{N}}^{\operatorname{Mp}(n)} \chi_{q, r}$ and $I_{n}(q, r)=\operatorname{Ind}_{M A \bar{N}}^{\operatorname{Mp}(n)} \pi_{q, r}$.

\section{NONCOMPACT PICTURES}

The noncompact realization of these induced representations amounts to restricting the functions $\phi$ to the copy of $\mathbb{R}^{n} \times \operatorname{Sym}(n, \mathbb{R})$ in $G$ given by the exponential of the nilpotent radical of the opposite parabolic to $\overline{\mathfrak{p}}_{\mathfrak{s p}}$ in $\mathfrak{s p}(n, \mathbb{R})$ and the corresponding complementary invariant abelian subalgebra to $\mathfrak{w}$ in $\mathfrak{h}_{2 n+1}$. We write

$$
I^{\prime}(q, r, s) \subsetneq \mathcal{C}^{\infty}\left(\mathbb{R}^{n} \times \operatorname{Sym}(n, \mathbb{R})\right)
$$

for the resulting representation. When necessary, we will coordinatize $\operatorname{Sym}(n, \mathbb{R})$ as $\mathbb{R}^{n(n+1) / 2}$ by writing $B=\left(t_{i j}\right)$ with $t_{i j}=t_{j, i}$.

It is straightforward to check that for $f \in I^{\prime}(q, r, s)$, the action of $g=\left(\left(\begin{array}{cc}A & B \\ C & D\end{array}\right), \varepsilon\right)$ in $\operatorname{Mp}(n)$ on $f$ is given by

$$
\begin{aligned}
((g, \varepsilon) \cdot f)(x, t)=i^{l q}|\operatorname{det}(A-t C)|^{r} e^{-s x C(A-t C)^{-1} x^{T}} & \\
\cdot & f\left(x\left(-C^{T} t+A^{T}\right)^{-1},(A-t C)^{-1}(t D-B)\right)
\end{aligned}
$$

when $\operatorname{det}(A-t C) \neq 0$ and where $l \in \mathbb{Z}_{4}$ satisfies

$$
\varepsilon\left(g^{-1} \cdot t\right)=i^{l}|\operatorname{det}(A-t C)|^{-1 / 2} .
$$

For $h=\left(x_{0}, y_{0}, z_{0}\right) \in \mathbb{R}^{n} \times \mathbb{R}^{n} \times \mathbb{R}$, the action of $h \in H_{2 n+1}$ on $f$ is given by $(h \cdot f)(x, t)=e^{s\left(2 x x_{0}^{T}+z_{0}-x_{0} t x_{0}^{T}-y_{0} x_{0}^{T}\right)} \cdot f\left(x-y_{0}-x_{0} t, t\right)$.

In a similar fashion, we also have the noncompact realizations of $I(q, r)$ and $I_{n}(q, r)$ given by restriction to $N \cong \operatorname{Sym}(n, \mathbb{R})$. We denote these realizations by $I^{\prime}(q, r) \subseteq \mathcal{C}^{\infty}(\operatorname{Sym}(n, \mathbb{R}))$ and $I_{n}^{\prime}(q, r) \subseteq \mathcal{C}^{\infty}\left(\operatorname{Sym}(n, \mathbb{R}), \mathbb{C}^{n}\right)$. There are $\operatorname{Mp}(n)$-intertwining maps

$$
\mathcal{E}: I^{\prime}(q, r, s) \rightarrow I^{\prime}(q, r), \mathscr{E}_{n}: I^{\prime}(q, r, s) \rightarrow I_{n}^{\prime}\left(q, r-n^{-1}\right)
$$

given by mappings $f \rightarrow f(0, \cdot)$ and $f \rightarrow \nabla f(0, \cdot)$, respectively. Using the noncompact realization, a Lie algebra calculation shows the following.

Theorem 1. For $r=-1 / 2$, the set of functions $f \in I^{\prime}(q, r, s)$ satisfying the system of partial differential equations from system $(*)$ is $G$-invariant. 
For the rest of the paper, we assume $r=-1 / 2$. Now let $s=i \sigma \neq 0$ be purely imaginary and write $\varepsilon_{\sigma}=\operatorname{sgn}(\sigma)$. Define

$$
\mathscr{D}^{\prime} \subseteq I^{\prime}(q, r, s) \subseteq \mathcal{C}^{\infty}\left(\mathbb{R}^{n} \times \operatorname{Sym}(n, \mathbb{R})\right)
$$

to be the space of functions $f \in I^{\prime}(q, r, s)$ that satisfy the system of partial differential equations from system $(*)$ with $f(\cdot, 0) \in \delta\left(\mathbb{R}^{n}\right)$, a Schwartz function. Write $\mathscr{D}_{+}^{\prime}$ and $\mathscr{D}_{-}^{\prime}$ for the functions in $\mathscr{D}^{\prime}$ that are even (odd) in $x$ for each $t \in \operatorname{Sym}(n, \mathbb{R})$. A nontrivial argument using Fourier analysis shows the following.

Theorem 2. The space $\mathscr{D}^{\prime}$ is $G$-invariant and, for $q=-\operatorname{sgn} \sigma, \mathscr{D}_{ \pm}^{\prime}$ is nonzero.

For example, when $\sigma>0$ and $q \equiv-1, f_{+} \in \mathscr{D}_{+}^{\prime}$ with

$$
f_{+}(x, t)=\varepsilon_{t}\left(i I_{n}\right)^{-1} e^{-\sigma x\left(I_{n}+i t\right)^{-1} x^{T}},
$$

where $\varepsilon_{t}(Z)$ is the analytic continuation of the function $Z \mapsto \sqrt{\operatorname{det}\left(I_{n}+t Z\right)}$ to $Z \in \mathfrak{H}_{n}$ for sufficiently small $Z$. Similarly, for $\alpha \in \mathbb{C}^{n}, f_{+, \alpha} \in \mathscr{D}_{-}^{\prime}$ where

$$
f_{+, \alpha}(x, t)=\varepsilon_{t}\left(i I_{n}\right)^{-1}\left(x\left(I_{n}+i t\right)^{-1} \alpha^{T}\right) e^{-\sigma x\left(I_{n}+i t\right)^{-1} x^{T}} .
$$

When $\sigma<0$ and $q \equiv 1, f_{-}$and $f_{-, \alpha}$ can be defined using complex conjugation.

\section{RESTRICTION TO $t=0$}

In order to guarantee the existence of nontrivial solutions in $\mathscr{D}^{\prime}$, we assume $q=$ $-\operatorname{sgn} \sigma$ for the rest of the paper. By Fourier analysis, the map from $\mathscr{D}^{\prime}$ to $\delta\left(\mathbb{R}^{n}\right)$ given by restriction to $t=0$ is injective. Following this map with the Fourier transform gives the injective map: $\mathscr{E}: \mathbb{D}^{\prime} \rightarrow \delta\left(\mathbb{R}^{n}\right)$ defined by $(\varepsilon f)(x)=\hat{f}(x, 0)$. We also write $\delta=\operatorname{Im}(\mathscr{E})$ and $\delta_{+}$and $\delta_{-}$for the images of $\mathscr{D}_{+}^{\prime}$ and $\mathscr{D}_{-}^{\prime}$, respectively. We make $\delta$ into a $G$-module by requiring $\mathcal{E}$ to be an intertwining isomorphism. Finally, consider the dilation operator defined by $(T f)(x)=f\left(|\sigma|^{1 / 2} x / \pi \sqrt{2}\right)$. Making $T$ into an intertwining map, we get an equivalent action on $T(8) \subseteq \&\left(\mathbb{R}^{n}\right)$. Using standard analysis arguments and the definitions, we get the following result.

Theorem 3. If $s=i \sigma$ with $\sigma<0, T(8)$ is a dense $\mathrm{Mp}(n)$-invariant subspace in the oscillator representation. If $\sigma>0, T(8)$ is isomorphic to the dual to the oscillator representation.

In either case, this action completes to a unitary representation on $L^{2}\left(\mathbb{R}^{n}\right)$ and decomposes as a direct sum of irreducible representations via the set of odd and even functions, $L^{2}\left(\mathbb{R}^{n}\right)=L^{2}\left(\mathbb{R}^{n}\right)_{+} \oplus L^{2}\left(\mathbb{R}^{n}\right)_{-}$.

\section{RESTRICTION TO $x=0$}

Recall that we have an $\operatorname{Mp}(n)$-intertwining map $\mathscr{E}: I^{\prime}(q, r, s) \rightarrow I^{\prime}(q, r)$ given by $(\mathscr{E} f)(t)=f(0, t)$ and an intertwining map $\mathscr{E}_{n}: I^{\prime}(q, r, s) \rightarrow I_{n}^{\prime}\left(q, r-\frac{1}{n}\right)$ given by $\left(\mathscr{E}_{n} f\right)(t)=\nabla f(0, t)$. By restricting to $\mathscr{D}^{\prime}$ and pre-composing with $\mathcal{E}^{-1}$, 
we obtain $\operatorname{Mp}(n)$-maps $\mathscr{H}: \delta \rightarrow I^{\prime}(q, r)$ and $\mathscr{H}_{n}: \delta \rightarrow I_{n}^{\prime}\left(q, r-n^{-1}\right)$. A Fourier analysis shows they are given by the formulas

$$
\begin{aligned}
(\mathscr{H} f)(t) & =\int_{\mathbb{R}^{n}} f(\xi) e^{\frac{\pi^{2}}{s} \xi t \xi^{T}} d \xi \\
\left(\mathscr{H}_{n} f\right)(t) & =\left.\nabla\left(\int_{\mathbb{R}^{n}} f(\xi) e^{\frac{\pi^{2}}{s} \xi t \xi^{T}} e^{2 \pi i \xi x^{T}} d \xi\right)\right|_{x=0} .
\end{aligned}
$$

Clearly, $\boldsymbol{s}_{-} \subseteq \operatorname{ker} \mathscr{H}$ and $\boldsymbol{s}_{+} \subseteq \operatorname{ker} \mathscr{H}_{n}$ (equivalently, $\mathscr{D}_{-}^{\prime} \subseteq \operatorname{ker} \mathscr{\mathscr { S }}$ and $\mathscr{D}_{+}^{\prime} \subseteq$ ker $\left.\mathscr{S}_{n}\right)$. Showing that these are the entire kernels involves inverting $\left.\mathscr{H}\right|_{\boldsymbol{s}_{+}}$and $\left.\mathscr{H}_{n}\right|_{\boldsymbol{S}_{-}}$(equivalently, $\mathscr{E}_{\mathscr{D}_{+}^{\prime}}$ and $\left.\mathscr{E}_{n}\right|_{\mathscr{D}_{-}^{\prime}}$ ). A straightforward Fourier analysis requires a bit more care due to the fact that the images usually do not have sufficient decay properties to be $L^{1}$ or $L^{2}$ functions (unless $n=1$, see [22]). In fact, if we could view $f \in \mathscr{D}^{\prime} \subseteq I^{\prime}(q, r, s)$ as a tempered distribution $f(x, \cdot) \in \mathcal{S}^{\prime}(\operatorname{Sym}(n, \mathbb{R}) \cong$ $\mathbb{R}^{n(n+1) / 2}$ ) and writing $\mathcal{F}$ for the Fourier transform on $\delta(\operatorname{Sym}(n, \mathbb{R}))$ given by $(\mathscr{F} f)(\tau)=\int_{\operatorname{Sym}(n, \mathbb{R})} f(t) e^{-2 \pi i \operatorname{tr}(t \tau)} d t$, it would be possible to show that $\mathscr{F} f$ is supported on $\left\{\tau \in \operatorname{Sym}(n, \mathbb{R}) \mid \tau_{i, i} \tau_{j, j}=\tau_{i j}^{2}\right.$ all $\left.i \neq j\right\}$. This is, of course, a rank of at most one condition on $\operatorname{Sym}(n, \mathbb{R})$.

As a result, it will be useful to consider the cone defined by the function $\theta$ : $\mathbb{R}^{n} \rightarrow \operatorname{Sym}(n, \mathbb{R})$ given by $\theta(y)=\pi y^{T} y / 2 \sigma$. For $f \in D^{\prime} \subseteq I^{\prime}(q, r, s)$ and each $x \in \mathbb{R}^{n}$, it is possible to show that $f(x, \cdot)$ may be viewed as a tempered distribution on $\operatorname{Sym}(n, \mathbb{R})$ given by

$$
\langle f(x, \cdot), \phi\rangle=\int_{\operatorname{Sym}(n, \mathbb{R})} f(x, t) \phi(t) d t
$$

for each $\phi \in \mathcal{S}(\operatorname{Sym}(n, \mathbb{R}))$. Its Fourier transform $\mathcal{F} f(x, \cdot) \in \mathcal{S}^{\prime}(\operatorname{Sym}(n, \mathbb{R}))$ is then shown to be $\langle\mathcal{F} f(x, \cdot), \phi\rangle=\int_{\mathbb{R}^{n}} \hat{f}(\xi, 0)(\phi \circ \theta)(\xi) e^{2 \pi i \xi x^{T}} d \xi$ and is supported on $\operatorname{Im} \theta$. Similarly, for each $1 \leq j \leq n, \partial_{x_{j}} f(x, \cdot)$ may be viewed as a tempered distribution on $\operatorname{Sym}(n, \mathbb{R})$ given by

$$
\left\langle\partial_{x_{j}} f(x, \cdot), \phi\right\rangle=\int_{\operatorname{Sym}(n, \mathbb{R})} \partial_{x_{j}} f(x, t) \phi(t) d t
$$

for an arbitrary $\phi \in \delta(\operatorname{Sym}(n, \mathbb{R}))$. Its Fourier transform is then shown to be $\mathcal{F}\left(\partial_{x_{j}} f\right)(x, \cdot) \in \mathcal{S}^{\prime}(\operatorname{Sym}(n, \mathbb{R}))$ given by

$$
\left\langle\mathcal{F}\left(\partial_{x_{j}} f\right)(x, \cdot), \phi\right\rangle=2 \pi i \int_{\mathbb{R}^{n}} \xi_{j} \hat{f}(\xi, 0)(\phi \circ \theta)(\xi) e^{2 \pi i \xi x^{T}} d \xi
$$

and is also supported on $\operatorname{Im} \theta$.

Again, using Fourier analysis, it is then possible to show that $\left.\mathscr{H}\right|_{\boldsymbol{s}_{+}}$is injective and $\left.\mathscr{H}_{n}\right|_{\boldsymbol{s}_{-}}$is injective (equivalently, $\mathscr{G}_{\mathscr{D}_{+}^{\prime}}$ is injective and $\left.\mathscr{G}_{n}\right|_{\mathscr{D}_{-}^{\prime}}$ is injective). If we write $\mathcal{l}_{ \pm}^{\prime}$ for the image of $\mathscr{D}_{ \pm}^{\prime}$ under $\mathscr{G}$ and $\mathscr{E}_{n}$, respectively (alternately, the image of $\delta_{ \pm}$under $\mathscr{H}_{\text {and }} \mathscr{H}_{n}$, respectively), and embed $\delta \hookrightarrow \delta^{\prime}(\operatorname{Sym}(n, \mathbb{R}))$ 
via $\theta$ by mapping $\psi \rightarrow\langle\psi, \cdot\rangle$ where $\langle\psi, \phi\rangle=\int_{\mathbb{R}^{n}} \psi(\xi)(\phi \circ \theta)(\xi) d \xi$ and embed $\delta \hookrightarrow \delta^{\prime}\left(\operatorname{Sym}(n, \mathbb{R}), \mathbb{R}^{n}\right)$ via $\theta$ by mapping $\psi \rightarrow\langle\psi, \cdot\rangle$, where

$$
\langle\psi, \phi\rangle=\left(\int_{\mathbb{R}^{n}} \xi_{j} \psi(\xi)(\phi \circ \theta)(\xi) d \xi\right)_{j=1, \ldots, n}
$$

for $\phi \in S(\operatorname{Sym}(n, \mathbb{R}))$, we can prove:

Theorem 4. The spaces $d_{ \pm}^{\prime} \subseteq I^{\prime}(q, r)$ are given explicitly by

$$
\begin{aligned}
& d_{+}^{\prime}=\left\{\mathscr{F} \psi \mid \psi \in \delta \subseteq \mathcal{S}^{\prime}(\operatorname{Sym}(n, \mathbb{R}))\right\}, \\
& d_{-}^{\prime}=\left\{\mathscr{F} \psi \mid \psi \in \delta \subseteq \mathcal{S}^{\prime}\left(\operatorname{Sym}(n, \mathbb{R}), \mathbb{R}^{n}\right)\right\} .
\end{aligned}
$$

\section{7. $K$-FINITE VECTORS}

If $M \in M_{n}(\mathbb{C})$ and $p$ is a complex valued polynomial on $\mathbb{R}^{n}$, define $\tilde{p}(x, M)$ by

$$
\tilde{p}(x, M)=e^{|\sigma| x M x^{T}} p\left(\partial_{x}\right)\left(e^{-|\sigma| x M x^{T}}\right),
$$

with $p\left(\partial_{x}\right)$ representing the constant coefficient differential operator obtained by replacing $x_{j}$ by $\partial_{x_{j}}$. For $p$ of the form $x^{\alpha}, \tilde{p}$ defines a generalization of the Hermite polynomials. Making use of the previous theorems and knowledge of the oscillator representation, we get the following description of $K$-finite vectors.

Theorem 5. Up to constant multiples, the highest $(\sigma<0)$ and lowest $(\sigma>0)$ $K$-finite vectors of $\left(\mathscr{D}_{+}^{\prime}\right)_{K}$ are given by the functions $f_{-}$and $f_{+}$, respectively.

The highest and lowest $K$-type vectors of $\left(D_{-}^{\prime}\right)_{K}$ consist of the functions $f_{-, a}$ and $f_{+, a}$, respectively, for $a \in \mathbb{C}^{n}$.

In general, the $K$-finite vectors in $\mathscr{D}^{\prime}$ consist of the functions $f_{-, p}$ and $f_{+, p}$, where

$$
f_{-, p}(x, t)={\overline{\varepsilon_{t}\left(i I_{n}\right)}}^{-1} \tilde{p}\left(x,\left(I_{n}-i t\right)^{-1}\right) e^{\sigma x\left(I_{n}-i t\right)^{-1} x^{T}}
$$

and

$$
f_{+, p}(x, t)=\varepsilon_{t}\left(i I_{n}\right)^{-1} \tilde{p}\left(x,\left(I_{n}+i t\right)^{-1}\right) e^{-\sigma x\left(I_{n}+i t\right)^{-1} x^{T}},
$$

$p$ being a complex valued polynomial on $\mathbb{R}^{n}$.

Corollary 1. The spaces of the highest $(\sigma<0)$ and lowest $(\sigma>0) K$-finite vectors of $\left(d_{+}^{\prime}\right)_{K}$ are spanned by the functions $f_{\mathrm{sgn} \sigma} \sigma$ given by $f_{-}(0, t)=\overline{\varepsilon_{t}\left(i I_{n}\right)}-1$ and $f_{+}(0, t)=\varepsilon_{t}\left(i I_{n}\right)^{-1}$, respectively. The highest $(\sigma<0)$ and lowest $(\sigma>0)$ $K$-type vectors of $\left(d_{-}^{\prime}\right)_{K}$ are given by the functions $f_{\operatorname{sgn} \sigma, a}$, where

$$
f_{-, a}(t)={\overline{\varepsilon_{t}\left(i I_{n}\right)}}^{-1} a\left(I_{n}-i t\right)^{-1}
$$


and

$$
f_{+, a}(t)=\varepsilon_{t}\left(i I_{n}\right)^{-1} a\left(I_{n}+i t\right)^{-1}
$$

for $a \in \mathbb{R}^{n}$.

\section{REFERENCES}

[1] S. Ali and M. Englis, "Quantization methods: a guide for physicists and analysts," Rev. Math. Phys., vol. 17, no. 4, pp. 391-490, 2005.

[2] S. Berceanu, AIP Conf. Proc., ser. Geometric methods in physics. Melville, NY: Amer. Inst. Phys., 2008, vol. 1079, ch. Generalized squeezed states for the Jacobi group, pp. 67-75.

[3] S. Berceanu and A. Gheorghe, "On the geometry of Siegel-Jacobi domains," Int. J. Geom. Methods Mod. Phys., vol. 8, no. 8, pp. 1778-1798, 2011.

[4] R. Bernt, "The Heat Equation and Representations of the Jacobi Group," Contemp. Math., vol. 390, pp. 47-68, 2006.

[5] R. Bernt and R. Schmidt, Elements of the Theory of the Jacobi Group. Basel: Birkhäuser, 1998.

[6] D. Bump, Automorphic Forms and Representations, ser. Cambridge Studies in Advanced Mathematics. Cambridge University Press, 1998, vol. 55.

[7] M. Dineykhan, G. Ganbold, and G. V. Efimov, Oscillator representation in quantum physics, ser. Lecture Notes in Phys. Berlin: Springer, 1995, vol. 26.

[8] M. Eichler and D. Zagier, The Theory of Jacobi Forms. Boston: Birkhäuser, 1985.

[9] T. Enright and R. Parthasarathy, Non Commutative Harmonic Analysis and Lie Groups, ser. Lecture Notes in Mathematics. Springer-Verlag, 1981, vol. 880, ch. A proof of a conjecture of Kashiware and Vergne, pp. 74-90.

[10] G. Folland, Harmonic Analysis in Phase Space, ser. Annals of Mathematics Studies. Princeton, New Jersey: Princeton University Press, 1989, vol. 122.

[11] V. Guillemin and S. Sternberg, "Geometric Quantization and Multiplicities of Group Representations," Invent. Math., vol. 67, pp. 515-538, 1982.

[12] V. Guillemin and S. Sternberg, Symplectic Techniques in Physics. Cambridge University Press, 1984.

[13] H. He, "Functions on symmetric spaces and oscillator representation," J. Funct. Anal., vol. 244, pp. 536-564, 2007.

[14] M. Hunziker, M. Sepanski, and R. Stanke, "The minimal representation of the conformal group and classical solutions to the wave equation," J. Lie Theory, vol. 22, pp. 301-360, 2012.

[15] J. I. Igusha, Theta Functions. Berlin: Springer, 1972.

[16] M. Kashiwara and M. Vergne, "On the Segal-Shale-Weil representations and harmonic polynomials," Invent. Math., vol. 44, no. 1, pp. 1-47, 1978.

[17] G. Lion and M. Vergne, The Weil Representation, Maslov Index and Theta Series, ser. Progress in Mathematics. Boston: Birkhäuser, 1980, vol. 6.

[18] D. Mumford, Tata Lectures on Theta I-III, ser. Progress in Mathematics. Boston: Birkhäuser, 1983, 1984, 1991, vol. 28, 43, 97.

[19] P. Olver, Applications of Lie Groups to Differential Equations, ser. Graduate Texts in Mathematics. New York: Springer, 1993, vol. 107.

[20] I. Segal, "Foundations of the theory of dynamical systems of infinitely many degrees of freedom, I," Mat. Fys. Medd. Danske Vid. Selsk., vol. 31, no. 12, 1959.

[21] M. Sepanski and R. Stanke, "On global $S L(2, \mathbb{R})$ symmetries of differential operators," J. Funct. Anal., vol. 224, no. 1, pp. 1-21, 2005.

[22] M. Sepanski and R. Stanke, "Global Lie symmetries of the heat and Schrödinger equation," J. Lie Theory, vol. 20, no. 3, pp. 543-580, 2010. 
[23] D. Shale, "Linear symmetries of free boson fields," Trans. Amer. Math. Soc., vol. 103, pp. 149167, 1962.

[24] D. Simms and N. Woodhouse, Lectures in Geometric Quantization, ser. Lecture Notes in Physics. Berlin-New York: Springer-Verlag, 1976, vol. 53.

[25] M. Vergne, First European Congress of Mathematics, ser. Progress in Mathematics. Basel: Birkhäuser, 1994, vol. 3, ch. Geometric quantization and equivariant cohomology, pp. 249-298.

[26] N. Wallach, Symplectic Geometry and Fourier Analysis, with an Appendix on Quantum Mechanics by Robert Herman, ser. Lie Groups: History, Frontiers and Application. Brookline, Mass.: Math Sci Press, 1977, vol. V.

[27] A. Weil, "Sur certaines groupes d'opérateurs unitaries," Acta Math., vol. 111, pp. 143-211, 1964.

[28] N. Woodhouse, Geometric Quantization, 2nd ed., ser. Oxford Mathematical Monographs, Oxford Science Publications. New York: The Clarendon Press, Oxford University Press, 1992.

Authors' addresses

\section{Markus Hunziker}

Department of Mathematics, Baylor University, Waco, TX 76798-7328

E-mail address: Markus_Hunziker@baylor.edu

\section{Mark R. Sepanski}

Department of Mathematics, Baylor University, Waco, TX 76798-7328

E-mail address: Mark_Sepanski@baylor.edu

\section{Ronald J. Stanke}

Department of Mathematics, Baylor University, Waco, TX 76798-7328

E-mail address: Ronald_Stanke@baylor. edu 